\title{
Numerical simulation and verification of weight function of electromagnetic flow meter with non-insulation surface pipe
}

\author{
Xuejing $\mathrm{Li}^{1, \text { a }}$ \\ ${ }^{1}$ School of Information Science and Technology, Sanda University, Shanghai 201209, China; \\ axuejingli99@outlook.com
}

Keywords: electromagnetic flow meter, non-insulation pipe surface, weight function, COMSOL Multiphysics

\begin{abstract}
Weight function of electromagnetic flow meter with non-insulation pipe is numerical simulated and verified. Weight function is very important for solution of flow velocity of distribution. In this paper, finite element method (FEM) is used for solving the weight function of this novel electromagnetic flow meter. First of all, numerical simulation model of electromagnetic flow meter with non-insulation surface pipe is built by the Comsol Multiphysics software. Boundary is researched. Secondly, weight values of different kinds of non-insulation pipe electromagnetic flow meters are analysis. These non-insulation pipe electromagnetic flow meters have different conductivity surface pipes and thicknesses. At lastly, induction electromotive force of electrodes is proved in the result of this paper. The result of distribution of virtual current is the same as weight value distribution has been proved under situation of uniform magnetic field. This method can be used in analysis different weight functions in other fields like prolusion of surface pipes and electrode poles of electromagnetic flow meter. It is very meaningful in medical and industrial field.
\end{abstract}

\section{Introduction}

Based on the Faraday's electromagnetic induction theory, Electromagnetic Flow Meter (EMFM) has been widely used in flow measurement field[1]. Weight function is very important and absolutely necessary for analysis on flow meter. It shows that each point at cross-section contribute to electrodes signals is different. And it follows the certain rule[2][3].

Traditional electromagnetic flow meter has insulation pipe or a layer of insulating material in the inner wall of the pipe[4]. Analytical solution of weight function shows value of center is 1 , value of circle (except electrodes) is 0.5 , the maximum value at electrodes[5]. In many conditions, the surface of pipe is not insulation or becoming non-insulation because of pollution. While, researching on weight function of EMFM with non-insulation surface pipe is seldom. It introduces some problems. Such as, measuring blood velocity using EMFM in medical, researching the effect of measure results because of the pipe or the electrodes are polluted.

This paper points out the difficulty of solving weight function of EMFM with non-insulation surface pipe is electrical conductivity of pipe should be considered. Traditional method of analyzing weight function is solving Laplace equation by means of Green's function. For solving weight value distribution of EMFM with non-insulation surface of pipe, virtual current density distribution is researched firstly. Changing of induction electromotive force between electrodes is pointed out. The analysis method put forward in this paper would solve the difficulties in medical and industrial field.

The most important thing is that the electrodes of the novel EMFM need not embedded in pipe, in another word is that the electrodes is not fixed on a certain location. However electrodes are on the outside of the pipe. The design in the paper makes collecting singles easily.

\section{Mold of EMFM with non-insulation pipe of surface}

EMFM in this paper abstracted as a cylindrical cavity body with non-insulation outer. Inner diameter is $R_{2}$, outer diameter is $R_{1}\left(R_{2}<R_{1}\right)$, pipe wall thickness is $h$. Smaller cylindrical cavity 
(diameter is $R_{2}$ ) filled with liquid of conductivity ${ }^{\sigma_{2}}$, pipe conductivity is ${ }^{\sigma_{1}}$. Establishing coordinate system, the origin point located in the circle center, the electrodes located in Y axis, geometry as Fig.1Two electrodes locate at the outside wall of the cavity. It is the advantage of the novel EMFM.

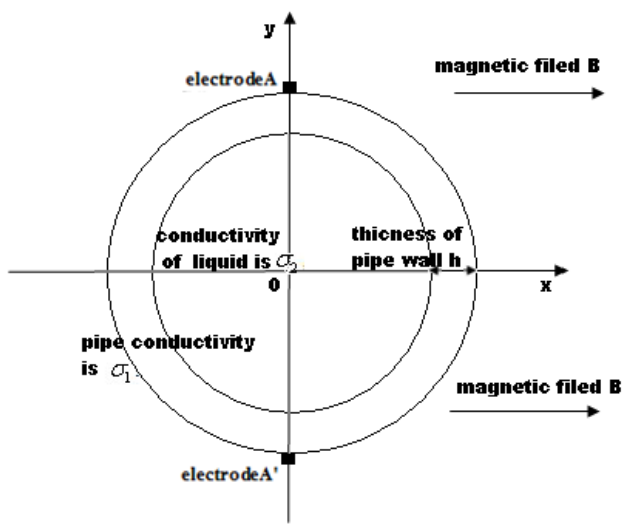

Fig. 1 Schematic diagram of EMFM with non-insulation pipe wall

\section{Analysis on the weight function of EMFM with non-insulation surface pipe}

The Green's function is used to solve the Laplace's equation. It is the method to obtain weight function of EMFM with insulation pipe surface [6]. Ohm's law is:

$\vec{j}=\sigma(\vec{E}+\vec{V}+\vec{B})$

If the displacement current is ignored:

$\nabla \cdot \vec{j}=0$

Substituting the equation (1) into the equation (2), we can get:

$$
\sigma\{\nabla \cdot \vec{E}+\nabla \cdot(\vec{V} \times \vec{B})\}+\nabla \sigma \cdot(\vec{E}+\vec{V} \times \vec{B})=0
$$

If the conductivity of the liquid is uniform:

$\nabla \sigma=0$

Using the Green's function gets the weight function analytical solution of EMFM (long pipe) with insulation pipe surface [2]

$$
w=\frac{R_{1}^{2}\left(R_{1}^{2}+x^{2}-y^{2}\right)}{R_{1}^{4}+2 R_{1}^{2}\left(x^{2}-y^{2}\right)+\left(x^{2}+y^{2}\right)^{2}}
$$

As EMFM with non-insulation pipe surface, equation (5) is untenable.

The reason is the conductive of the pipe is not equal with the conductive of the liquid..

It is difficult to solve equation (3) to get the weight function of EMFM with non-insulation surface pipe.

Virtual current density is decided by the shape of the electrodes and electrical boundary conditions of measurement pipe.

So the weight function is:

$W=\vec{B} \times \overrightarrow{j_{v}}$

The models in this paper, electrodes are point-electrodes.

So the virtual current of electrode is:

$\left.\frac{\partial G}{\partial r}\right|_{r=R}= \pm \frac{\delta}{R}$

Surface of the pipe wall is non-insulation, so the virtual current density in normal direction is not zero.

If virtual current density in electrode is $1\left(\mathrm{~A} / \mathrm{m}^{2}\right)$,

virtual current on inner wall point B (see in Fig.2) is $\vec{j}(0<\vec{j}<1)$,

It projects on non-insulation pipe wall is 
$\vec{j} \sin \alpha\left(\mathrm{A} / \mathrm{m}^{2}\right)$

As shown in the Fig.2, $\overrightarrow{B M}$ is the virtual current, $\overrightarrow{B N}$ is the tangent of circle by point B. Angle $\alpha$ between $\overrightarrow{B M}$ and $\overrightarrow{B N}$, So $\overrightarrow{M N}$ is Projection of $\overrightarrow{B M}$ in normal direction.

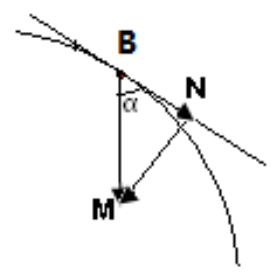

The Ohm's law:

Fig. 2 Schematic diagram of virtual current projection

$\vec{j}=\sigma_{1} \vec{E}$

Where $\sigma_{1}$ is the conductive of pipe. It shows that current density proportion to size of electric field.

Electric field intensity on point A is:

$\vec{E}=k \frac{\vec{Q}}{h^{2}}$

$k=\frac{1}{4 \varepsilon \pi} \quad \varepsilon=8.8542 \times 10^{12} \mathrm{Fm}^{-1}$

Where $h$ is the thickness of the pipe, $Q$ is the electric quantity of the electrode.

Substituting the equation (9) and the equation (10) into the equation (8):

$\vec{j}=\sigma_{1} k \frac{\vec{Q}}{h^{2}} \sin \alpha$

The equation (11) shows that the virtual current density is direct proportion to $\sigma_{1}$, inverse proportion to $h$.

$W=\vec{B} \times \sigma_{1} k \frac{\vec{Q}}{h^{2}} \sin \alpha$

Induced potential between $A$ and $A^{\prime}$ is:

$E_{A A^{\prime}}=\int_{V}(\vec{B} \times \vec{W}) \cdot \vec{v} d V$

$\bar{v}=\frac{1}{2} v_{\max }$

$v_{x}=v_{\max }\left[1-\left(\frac{r_{x}}{r}\right)^{2}\right]$

Where $\bar{v}$ is the average velocity, $v_{\max }$ is the liquid velocity on the center of the pipe, $r$ is the radius of the pipe, $r_{x}$ is the distance of central axis of the pipe on the radius direction. $v_{x}$ is the flow velocity on $r_{x}$.

If magnetic field is uniform, the velocity of flow is fixed. The change of weight function reflected as change of induction electromotive force between electrodes.

\section{Simulation of EMFM with non-insulation surface pipe}

The AC/DC module of COMSOL Multiphysics is used. Helmholtz coils are introduced to producing uniform magnetic fields. The flow field is laminar and the average velocity is $2 \mathrm{~m} / \mathrm{s}$. The radius of the pipe is $1 \mathrm{~m}$, and the electrodes are copper. The flow in the pipe is water $\left(25^{\circ} \mathrm{C}\right)$, and the conductive is $1.0 \mu \mathrm{s} / \mathrm{cm}$.

Fig. 3 shows the simulation results of the weight function of EMFM (three kinds conductive be choose: $1.035 \mathrm{e}^{-12} \mathrm{~s} / \mathrm{m}, 1.537 \mathrm{e}^{-7} \mathrm{~s} / \mathrm{m}, 4.5 \mathrm{e}^{-3} \mathrm{~s} / \mathrm{m}$ ). 


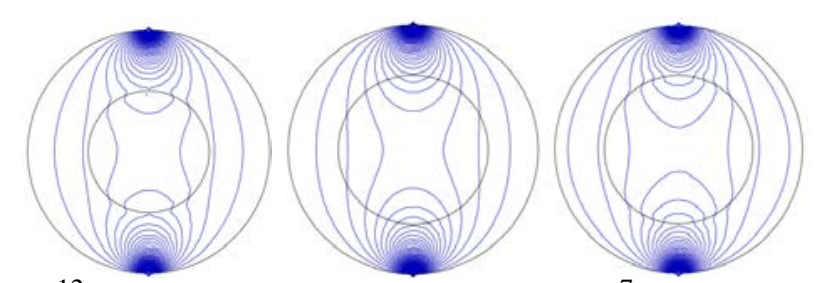

a. pipe conductive is $1.035 \mathrm{e}^{-12} \mathrm{~s} / \mathrm{mb}$. pipe conductive is $1.537 \mathrm{e}^{-7} \mathrm{~s} / \mathrm{m} c$. pipe conductive is $4.5 \mathrm{e}^{-3} \mathrm{~s} / \mathrm{m}$

Fig.3 Simulation diagram of weight function of EMFM

Fig.4 shows that change of conductive of pipe makes the change of weight function. Equation (13) is used to calculate induced electromotive force of electrodes. The result is shown in Fig. 4.

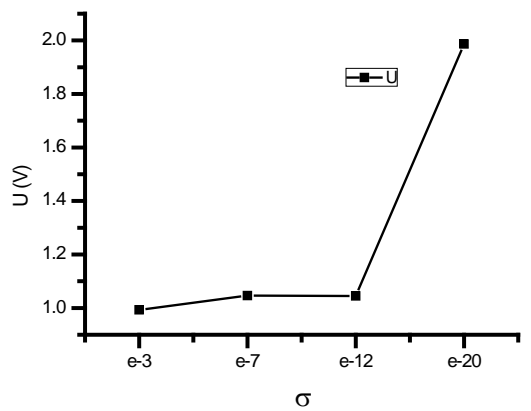

Fig.4 Curve of induced electromotive force

As can be seen from the Fig.4, the induced electromotive force decreased but the conductive of pipe is increased.

It also shows virtual current density changes with the conductive of the pipe. In another words, virtual current density is direct proportion with conductive of pipe. Equation (11) is verified.

\section{Summary}

The weight function shows the velocity of different location on across-section pipe contributes to different signals of the electrodes. The uniform weight function means every point has the same contribution. Low unevenness is very important to the design of EMFM. The virtual current density on juncture between non-insulation cavity and flow is $\sigma_{1} k \frac{\vec{Q}}{h^{2}} \sin \alpha$ can get a uniform weight function. The weight function of EMFM with non-insulation surface pipe changes with the conductive of pipe. The novel model of EMFM is very meaningful to solve problems according by pollution of electrodes and pipe surface in measurement. The novel EMFM with lots of non-insulation material surface pipes will play an important role in the future.

\section{References}

[1] COMSOL Corporation 2005 Femlab 3.2 User's Guide http://www.comsol.com.

[2] Shercliff J A. “Electromagnetic Flow Measurement”, Cambridge University Press, 1987.

[3] Torben Amby Christensen ,Morten Willatzen , "Investigating the effect of magnetic pipes connected to electromagnetic flowmeters using experimentally validated finite element models", Flow Measurement and Instrumentation, 21(2010)62-69.

[4] Bevir MK, “The theory of induced voltage electromagnetic flowmeters”, Journal of Fluid Mechanics, 43(1970) 577-590.

[5] J. Z. Wang, G.P. Lucas, G.Y. Tian, “A numerical approach to the determination of electromagnetic flow meter weight functions”, Meas. Sic. Technol, vol.18, pp.548-554, 2007.

[6] Roger C Baker, “On the concept of virtual current as a means to enhance verification of electromagnetic flowmeters,” Meas. Sci. Technol.22(2011) 1-10. 